\title{
ChemComm
}

\section{Long-term stability for cobalt-based dye- sensitized solar cells obtained by electrolyte optimization $\dagger$}

\author{
Cite this: Chem. Commun., 2014 \\ 50,6249 \\ Received 26th January 2014, \\ Accepted 23rd April 2014
}

DOI: $10.1039 / c 4 c c 00698 d$

www.rsc.org/chemcomm

\author{
Jiajia Gao, Muthuraaman Bhagavathi Achari and Lars Kloo*
}

\begin{abstract}
A significant improvement in the long-term stability for cobalt-based dye-sensitized solar cells (DSCs) under light-soaking conditions has been achieved by optimization of the composition of tris(2,2'-bipyridine) Co(II)/Co(III) electrolytes. The effects of component exchanges and changes were also studied during the optimization process.
\end{abstract}

Dye-sensitized solar cells (DSCs) have, since the groundbreaking work by O'Regan and Grätzel in 1991, been considered as a promising, green-energy technology. ${ }^{1}$ DSCs have showed both potential low-cost manufacturing and tunable structure properties in combination with comparably high power conversion efficiencies. $^{2,3}$ With the aim to avoid the shortcomings of the $\mathrm{I}^{-} / \mathrm{I}_{3}{ }^{-}$ system, with respect to light absorption and corrosivity, the recent search for alternative metal-organic redox systems has opened up a new direction of research; ${ }^{4}$ the to-date best system contains Co(II/III)-polypyridyl redox shuttles exhibiting over $12 \%$ conversion efficiency together with co-absorbed sensitizers. The improvement in conversion efficiency can be attributed to the more positive redox potential of the redox system, thus minimizing the overpotential required for sensitizer regeneration. ${ }^{5}$ Given a promising photovoltaic performance, the stability issue is, however, another challenge for cobalt-based DSCs before any commercial application can be considered. For DSCs as multi-component devices, there are inevitably various influential factors on the device stability; one decisive factor is the electrolyte, composed of redox shuttles, a selection of additives and the solvent.

As an excellent redox shuttle alternative, $\left[\mathrm{Co}(\mathrm{bpy})_{3}\right]^{2+/ 3+}$, was recently strongly questioned regarding the potential instability of $\left[\mathrm{Co}(\mathrm{bpy})_{3}\right]^{2+}$ with respect to ligand dissociation. ${ }^{6}$ For this reason, a new cobalt-based redox couple with a hexadentate ligand was designed and displayed a DSC durability of 100 hours under full solar irradiation. ${ }^{7}$ Acetonitrile, with its low viscosity

Division of Applied Physical Chemistry, Center for Molecular Devices, Department of Chemistry, KTH Royal Institute of Technology, SE-100 44, Stockholm, Sweden.E-mail: Larsa@kth.se

$\dagger$ Electronic supplementary information (ESI) available: Experiment details, toolbox data, $J-V$ characteristics data, EIS data and part of stability tests data. See DOI: 10.1039/c4cc00698d and high dielectric constant, is to date the best alternative as the electrolyte solvent of liquid DSCs from the perspective of device efficiency. However, conversion efficiency has to be balanced against device stability, due to the solvent"s potential drawback regarding volatility. Besides the search for high-boiling solvents, such as water, ${ }^{8} 3$-methoxy propionitrile (MPN) or ionic liquids, ${ }^{9}$ mixtures containing acetonitrile have also been investigated in order to identify systems that satisfy both requirements. Acetonitrile-ionic liquid mixture electrolytes were previously used for $\mathrm{I}^{-} / \mathrm{I}_{3}{ }^{-}$-based DSCs with comparable efficiency and excellent stability. ${ }^{10}$ Recently, Xiang et al. reported a gel electrolyte comprising a polymer and acetonitrile resulting in a highly efficient cobalt-based DSC with an outstanding durability under nearly $700 \mathrm{~h}$ of illumination. ${ }^{11}$ Additives, such as lithium salts and 4-tert-butyl pyridine (TBP), were proven to be highly necessary for high performance. The additives are invariably used together: $\mathrm{Li}^{+}$ ions expected to cause a lowering of the $\mathrm{TiO}_{2} \mathrm{CB}$ energy level, and this effect is often observed to offer an improvement in electron injection efficiency and electron lifetime. ${ }^{12}$ In contrast, employing TBP typically instead results in a negative shift of $\mathrm{TiO}_{2} \mathrm{CB}$ and as a consequence a reduced interfacial recombination loss and a relatively high open-circuit voltage. ${ }^{13}$ However, there are few papers addressing the influence of these additives on the durability of cobalt-based DSSCs, despite of an extensive relevant and discussion on $\mathrm{I}^{-} / \mathrm{I}_{3}{ }^{-}$-based systems. ${ }^{14-17}$ In an ongoing study in other solvent media, it has become clear that higher concentrations of the cobaltbased redox components, both the reduced and, surprisingly enough also, the oxidized forms offer better long-term stability with a small cost of lower initial conversion efficiency. In this work, a high and a low concentration of the cobalt-based redox system are investigated together with a series of control experiments regarding both TBP and $\mathrm{Li}^{+}$salt effects.

Even though a variety of strategies have been designed and tested for cobalt-based electrolyte systems with the aim to improve DSC stability, stability test results during long enough time (1000 h) and under standard light-soaking conditions (continuous 1 sun illumination and $60{ }^{\circ} \mathrm{C}$ temperature) are still lacking. This void in knowledge inspired us to optimize a highly stable and 


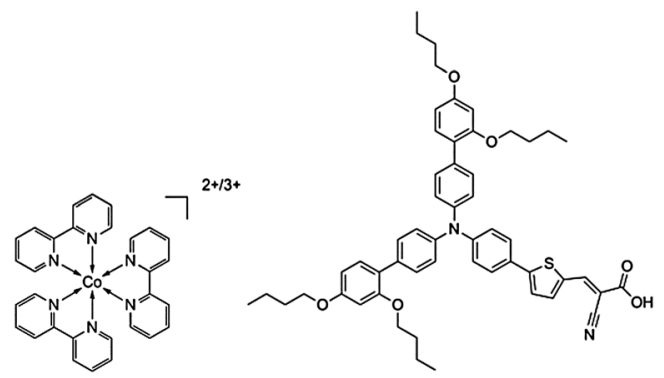

Fig. 1 Chemical structures of tris(2,2'-bipyridine) cobalt(II)/(III) complexes (left) and dye D35 (right).

efficient system, which in this work is achieved by an only slight changes with respect to the commonly used tris(2,2'-bipyridine) $\mathrm{Co}(\mathrm{II}) / \mathrm{Co}$ (III)-based electrolyte. Using the organic dye D35 as sensitizer (Fig. 1), such a system performed excellently over $1000 \mathrm{~h}$ in the standard stability test with only a small efficiency decline against the initial value.

In order to study the concentration-dependent influence on the device stability, electrolytes containing higher concentrations of $\mathrm{Co}(\mathrm{bpy})_{3}{ }^{2+}$ and $\mathrm{Co}(\mathrm{bpy})_{3}{ }^{3+}$ with the tetracyanoborate anion in acetonitrile with respect to the commonly-used cobalt electrolyte (AN-Lo-L; 0.22 M/0.05 M) were applied in D35-sensitized DSCs. The additives $\mathrm{LiClO}_{4}$ and TBP were initially in this study added in the same amounts as previously reported, respectively $0.1 \mathrm{M}$ and $0.2 \mathrm{M}$. All the DSCs were assembled using a $2.5-3 \mu \mathrm{m}$ thick, transparent, mesoporous $\mathrm{TiO}_{2}$ film, which has been proved to yield the highest photovoltaic performance in previous work. As shown by the preliminary stability test results shown in Fig. S1 (ESI $\dagger)$, there is a significant correlation between device stability and cobalt redox couple concentrations, and the optimal concentrations are based on the electrolyte AN-Hi-L (0.3 M/0.15 M). A stability test lasting 1000 hours under light-soaking conditions was further conducted for the resulting DSCs assembled with the champion electrolyte and the result is compared with that of electrolyte AN-Lo-L, shown in Fig. 2. The corresponding initial $I-V$ data are summarized in Table S1 (ESI $\dagger$ ). By comparison, DSCs incorporated with the electrolyte AN-Lo-L containing lower cobalt complex concentrations are clearly less stable; it can be linked to the faster degradation in open-circuit voltage $\left(V_{\mathrm{oc}}\right)$ and more obvious decline of fill factor (ff) arising at a later stage. The $V_{\mathrm{oc}}$ is determined by the difference between the quasi-Fermi level $\left(E_{\mathrm{F}, n}\right)$ of the $\mathrm{TiO}_{2}$ substrate and the redox potential of redox couple. $E_{\mathrm{F}, n}$ is dependent on the position of the $\mathrm{TiO}_{2}$ conduction band (CB) and the electron density in the $\mathrm{CB}$ and sub-bandgap trap states. Since the shift in redox potential is negligible the significant change in $V_{\text {oc }}$ can with a high probability be attributed to changes in the $E_{\mathrm{F}, n}$. This is also explained by results from toolbox measurements illustrated by the charge extraction as a function of open-circuit voltage, and electron lifetime as a function of charge extraction. As shown in Fig. S2(a) (ESI $\dagger$ ), in the early phase of the accelerated ageing test both DSCs containing the electrolytes $\mathbf{A N}-\mathbf{L o}-\mathbf{L}$ and $\mathbf{A N}-\mathbf{H i}-\mathbf{L}$ experienced a remarkable positive shift of the $\mathrm{TiO}_{2} \mathrm{CB}$ energy level with time, which is in agreement with the observed degradation in $V_{\mathrm{oc}}$ for both devices.
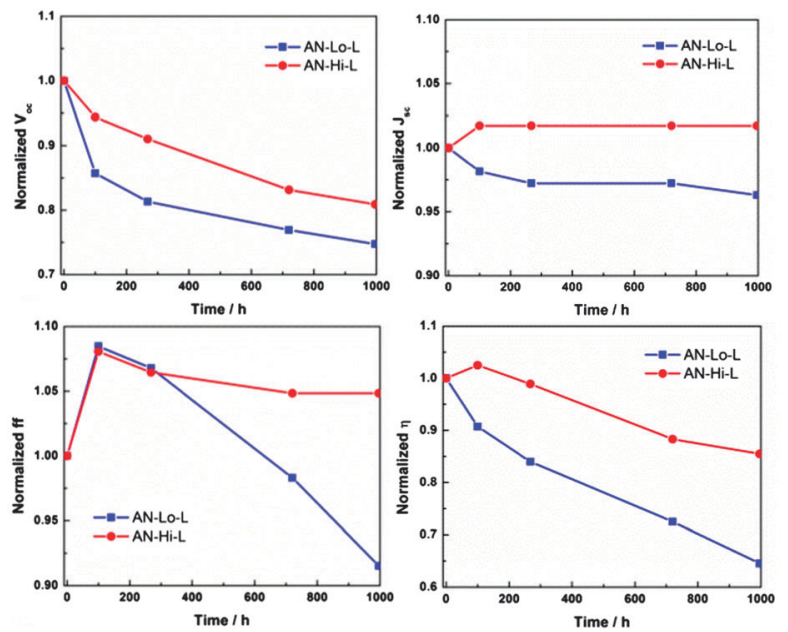

Fig. 2 Variation of photovoltaic parameters for DSCs based on the electrolytes AN-Lo-L (blue square) and $\mathbf{A N}-\mathbf{H i}-\mathbf{L}$ (red circle) during the stability test under light-soaking conditions ( 1 sun illumination, $60^{\circ} \mathrm{C}$ ).

However, for the electrolyte AN-Hi-L, containing the higher concentrations of the cobalt redox couple, the corresponding DSC exhibits a stable electron lifetime (Fig. S2(b), ESI $\dagger$ ), implying a relatively constant electron concentration in the $\mathrm{CB}$ of the $\mathrm{TiO}_{2}$ film. This explains the observed and significantly lower degradation rate of $V_{\text {oc }}$ with respect to that of DSC employing the electrolyte AN-Lo-L. Electrochemical impedance spectroscopy (EIS) measurements were also conducted in order to examine the change of characteristic device resistances during the test. It appears that DSCs containing lower concentrations of the cobalt redox couple suffer from more serious mass-transport limitation after long-term light soaking (Fig. S3(b and c), ESI $\dagger$ ), and consequently such devices show a faster drop in ff.

However, upon increasing cobalt complex concentrations, the risk of interfacial charge recombination is also increased, as observed and as expected (Fig. S3(a), ESI $\dagger$ ). To our knowledge, $\mathrm{Li}^{+}$ salts also give the same effect. Therefore, we further compared DSCs containing the electrolyte $\mathbf{A N}-\mathbf{H i}-\mathbf{L}$ and another similar electrolyte AN-Hi without $\mathrm{Li}^{+}$salt $\left(\mathrm{LiClO}_{4}\right)$ addition. Rather unexpectedly shown in Fig. 3, a better long-term stability was obtained for the device containing no additions of lithium salt, and also a comparable conversion efficiency after reaching its peak photocurrent

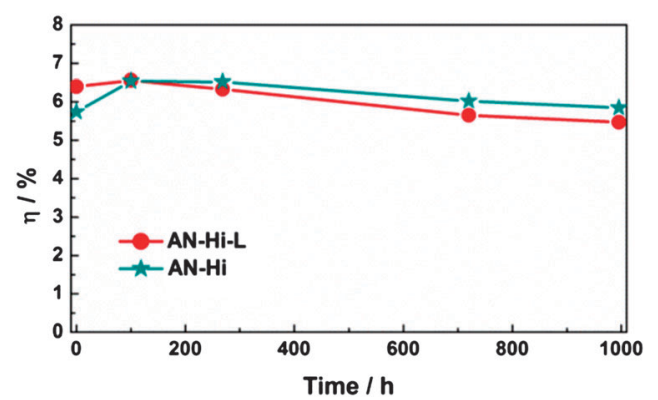

Fig. 3 Efficiency variation over time for DSCs based on the electrolytes $\mathbf{A N}-\mathbf{H i}-\mathbf{L}$ (red circle) and $\mathbf{A N}-\mathbf{H i}$ (cyan star) during the stability test under light-soaking conditions ( 1 sun illumination, $60^{\circ} \mathrm{C}$ ). 

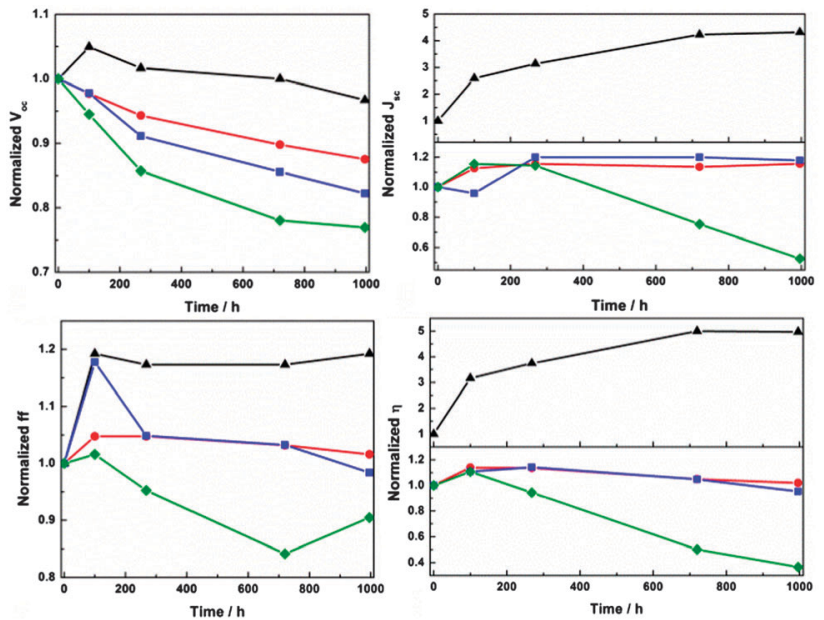

Fig. 4 Variation of photovoltaic parameters for DSCs based on a series of AN-Hi-type electrolytes containing different concentrations of TBP: $0 \mathrm{M}$ (black, triangle), 0.2 M (AN-Hi, red, circle), 0.5 M (blue, square) and 0.7 M (green, diamond).

(Fig. S4, ESI $\dagger$ ). The improvement can mainly be attributed to the slower decrease in $V_{\mathrm{oc}}$. As suggested by the results in Fig. S5(a and b) (ESI $\dagger$ ), this improvement can be explained by an increase in electron lifetime with time compensating for the loss caused by the positive shift of the $\mathrm{TiO}_{2}$ conduction band. In fact, the cells containing the AN-Hi electrolyte formally show a $4 \%$ better conversion efficiency after 1000 hours to light exposure at elevated temperature as compared to the initial value; however, the decline from the maximum efficiency after about 100 hours is about 8\% (though levelling off at longer exposure).

DSCs were also fabricated based on a series of AN-Hi-type electrolytes only varying the amount of added TBP from $0 \mathrm{M}$ to $0.2 \mathrm{M}, 0.5 \mathrm{M}, 0.7 \mathrm{M}$, respectively. By comparison of the initial performance after device fabrication (see Table S1, ESI $\dagger$ ), the considerable increase in all four photovoltaic parameters when increasing TBP concentration from $0 \mathrm{M}$ to $0.2 \mathrm{M}$ clearly indicates the key role of TBP for the devices. Stability tests under light-soaking conditions were subsequently conducted, and the results after 1000 hours are shown in Fig. 4. An increasingly rapid decline was observed in both $V_{\mathrm{oc}}$ and $J_{\mathrm{sc}}$, and consequently $\eta$, upon increasing the concentration of TBP further from $0.2 \mathrm{M}$ to $0.5 \mathrm{M}$ and $0.7 \mathrm{M}$. The change in interfacial recombination resistance $\left(R_{\mathrm{CT}}\right)$ of the corresponding DSCs, between electrons in the $\mathrm{TiO}_{2}$ film and oxidized species in electrolyte, is shown in Fig. S6 (ESI $\dagger$ ). It clearly indicates the two-side effect of TBP on these DSC devices: initially it well suppresses the interfacial recombination as commonly regarded but during the stability test, higher concentration of TBP induces a faster increase in the recombination loss, well in consistent with the faster $V_{\mathrm{oc}}$ degradation. The proposed reason for the adverse impact of TBP is that it as a relatively strong Lewis base induces dye desorption and thus a change in surface structure, which enhanced recombination losses; this effect may be accelerated by the light-soaking treatment. An unexpectedly continuous increase in $J_{\mathrm{sc}}$ is noted for DSCs without TBP added. Displaying a significantly stable $V_{\text {oc }}$ and ff, it seems to exhibit a surprising 'activation' process instead of degradation during the stability test. The underlying reasons are under investigation.

In conclusion, standard stability tests of $1000 \mathrm{~h}$ under lightsoaking conditions were conducted for DSCs containing the cobalt-acetonitrile-based electrolytes in this work. An impressive result of long-term durability was noted for this system; certainly not expected for the volatile solvent acetonitrile. The electrolyte composition has been demonstrated as the key factor for device stability. Minor adjustments involving a small increase in cobalt complex concentrations and the removal of a lithium-salt additive offered a significant improvement in device stability. TBP was, in line with numerous previous studies, identified as a highly important additive, however only in moderate concentrations.

The authors acknowledge the Swedish National Research Council, the Swedish Energy Agency as well as the China Scholarship Council (CSC) for financial support.

\section{Notes and references}

1 B. O'Regan and M. Grätzel, Nature, 1991, 353, 737-740.

2 P. Péchy, T. Renouard, S. M. Zakeeruddin, R. Humphry-Baker, P. Comte, P. Liska, L. Cevey, E. Costa, V. Shklover, L. Spiccia, G. B. Deacon, C. A. Bignozzi and M. Grätzel, J. Am. Chem. Soc., 2001, 123, 1613-1624.

3 A. Hagfeldt, G. Boschloo, L. Sun, L. Kloo and H. Pettersson, Chem. Rev., 2010, 110, 6595-6663.

4 J. Cong, X. Yang, L. Kloo and L. Sun, Energy Environ. Sci., 2012, 5, 9180-9194.

5 A. Yella, H.-W. Lee, H. N. Tsao, C. Yi, A. K. Chandiran, M. K. Nazeeruddin, E. W.-G. Diau, C.-Y. Yeh, S. M. Zakeeruddin and M. Grätzel, Science, 2011, 334, 629-634.

6 T. M. McCormick, Z. Han, D. J. Weinberg, W. W. Brennessel, P. L. Holland and R. Eisenberg, Inorg. Chem., 2011, 50, 10660-10666.

7 M. K. Kashif, M. Nippe, N. W. Duffy, C. M. Forsyth, C. J. Chang, J. R. Long, L. Spiccia and U. Bach, Angew. Chem., Int. Ed., 2013, 52, $5527-5531$.

8 W. Xiang, F. Huang, Y.-B. Cheng, U. Bach and L. Spiccia, Energy Environ. Sci., 2013, 6, 121-127.

9 D. Xu, H. Zhang, X. Chen and F. Yan, J. Mater. Chem. A, 2013, 1, 11933-11941.

10 Z. Yu, N. Vlachopoulos, A. Hagfeldt and L. Kloo, RSC Adv., 2013, 3, 1896-1901.

11 W. Xiang, W. Huang, U. Bach and L. Spiccia, Chem. Commun., 2013, 49, 8997-8999.

12 S. Nakade, Y. Makimoto, W. Kubo, T. Kitamura, Y. Wada and S. Yanagida, J. Phys. Chem. B, 2005, 109, 3488-3493.

13 T. M. Koh, K. Nonomura, N. Mathews, A. Hagfeldt, M. Grätzel, S. G. Mhaisalkar and A. C. Grimsdale, J. Phys. Chem. C, 2013, 117, 15515-15522.

14 S. Nakade, T. Kanzaki, S. Kambe, Y. Wada and S. Yanagida, Langmuir, 2005, 21, 11414-11417.

15 A. G. Kontos, T. Stergiopoulos, V. Likodimos, D. Milliken, H. Desilvesto, G. Tulloch and P. Falaras, J. Phys. Chem. C, 2013, 117, 8636-8646.

16 V. Likodimos, T. Stergiopoulos, P. Falaras, R. Harikisun, J. Desilvestro and G. Tulloch, J. Phys. Chem. C, 2009, 113, 9412-9422.

17 C. Zhang, Y. Huang, S. Chen, H. Tian, L. e. Mo, L. Hu, Z. Huo, F. Kong, Y. Ma and S. Dai, J. Phys. Chem. C, 2012, 116, 19807-19813. 